Central Washington University

ScholarWorks@CWU

All Faculty Scholarship for the College of the Sciences

7-24-2016

Comparison of formulations of applied tasks with intervals, fuzzy sets and probability approaches

Boris Kovalerchuk

Vladik Kreinovich

Follow this and additional works at: https://digitalcommons.cwu.edu/cotsfac

Part of the Theory and Algorithms Commons 


\title{
Comparison of formulations of applied tasks with intervals, fuzzy sets and probability approaches
}

\author{
Boris Kovalerchuk \\ Dept. of Computer Science, Central Washington University, \\ 400 E. University Way, Ellensburg, WA 98926, USA \\ Vladik Kreinovich \\ Dept. of Computer Science, University of Texas at El Paso \\ 500 W. University Avenue, El Paso, TX 79968, USA
}

\begin{abstract}
The focus of this paper is to clarify the concepts of solutions in linear equations in interval, probabilistic and fuzzy sets setting for real word tasks. There is a fundamental difference between formal definitions of the solutions and physically meaningful concept of solution in applied tasks when equations have uncertain components. For instance, a formal definition of the solution in terms of Moore interval analysis can be completely irrelevant for solving a real world task. We show that formal definitions must follow meaningful concept of the solution in the real world. The paper proposed several formalized definitions of the concept of solution for the linear equations with uncertain components in the interval, probability and fuzzy sets term.
\end{abstract}

Keywords: interval linear equations, fuzzy linear equations, stochastic linear equations, quantifiers, solutions of uncertain equations.

\section{Introduction}

Most of the work in fuzzy equations has been concern about algorithms and theorems for solving fuzzy linear equation under Zadeh's extension principle in exact and approximate setting, e.g., [Yager, 1979; Sanchez, 1984; Di Nola, Pedrycz,Sessa, 1985, Peeva, 1991, Buckley, Feuring, 2000, Horcık, 2008; Skalna et al, 2008]. The typical method to solve fuzzy equation is converting to a set of interval task with alpha-cuts [e.g., Skalna et al, 2008]. One work is standing out [Dubois, Prade, 1984], where the authors provided arguments and an example that show the need to go beyond the extension principle. The concepts of optimistic and pessimistic operations on fuzzy numbers have been proposed in this context. We use these productive concepts (with some modifications) to define the respective concepts of the optimistic and pessimistic solutions for uncertain equations.

The focus of this paper - which largely expands on [Kreinovich 2016] -- is to clarify the concepts of solutions of linear equations in interval, probabilistic, and fuzzy-set setting for real-world tasks. When equations have uncertain components in applied tasks, there is a fundamental difference between formal definitions of the solutions and a physically meaningful concept of solution. For instance, a formal definition of the solution in terms of Moore interval analysis can be completely irrelevant for solving a real-world task. We show that formal definitions must follow a meaningful concept of the solution in the real world, not another way around. The solution that is claimed to be a solution of the uncertain equation should be a solution of the real world task not a just a result of a formal mathematical definition. Hisdal [1988] worded this challenge in the following way: somebody proposed a solution now we need to find a problem for it.

The example of equation with uncertainty is

$$
\mathrm{A}+\mathrm{X}=\mathrm{B}
$$


where A, X and B are intervals, pdfs or fuzzy sets. The task is, given A and B, to find X. What is the sum of multiple dependent intervals, pdfs and fuzzy sets is not clear. The same is true for the pdf and fuzzy membership function for the sum. For instance, a sum of $\mathrm{x}+\mathrm{y}$ with respective pdfs $f(\mathrm{x})$ and $g(\mathrm{y})$ has a joint probability distribution $\mathrm{q}(\mathrm{x}, \mathrm{y})=\mathrm{f}(\mathrm{x})^{*} \mathrm{~g}(\mathrm{y} \mid \mathrm{x})$. If $\mathrm{F}$ and $\mathrm{G}$ are independent then $\mathrm{q}(\mathrm{x}, \mathrm{y})=\mathrm{f}(\mathrm{x}) * \mathrm{~g}(\mathrm{y})$. However in general we cannot make this independence assumption and the conditional probability $\mathrm{g}(\mathrm{y} \mid \mathrm{x})$ often is unknown, As a result equation (1) is illposed, it is not fully defined. In the fuzzy sets setting, a similar uncertainty is related to the selection of an aggregation operator.

\section{Tasks}

\subsection{Airport Task.}

Dubois and Prade [1984] formulated an applied example (in fuzzy sets setting) where they used concepts of optimistic and pessimistic operations. Below we consider their original task, as well as our generalization to formulate and compare precise point-based, interval-based, probability-based, and fuzzy sets-based formulations of what is a solution.

Airport Task. Person $\mathrm{P}$ wants to ensure that he will not miss a plane. His goal is to make sure he arrives at the airport by desired time $T_{A}$ (expressed precisely or with some uncertainty as an interval, as a probability, or a fuzzy set/number) in spite of imprecise duration D of his preceding activities such as wake up time, washing, eating breakfast, driving to the airport, etc.). Dubois and Prade formulated the goal as finding required wakeup time.

Later we will consider a more general goal. To clarify that we need to understand the problem when formalizing what is a solution, we will also consider a modification of the above task, in which we know that the person $\mathrm{P}$ arrived at the airport on time, and we want to find out when he/she woke up.

\subsection{Precise and Interval settings}

Consider first the precise setting where all data are known and precise. Let precise durations of all activities $d_{i}$ and the desired time to arrive to the airport $t_{A}$ be known. Then the required wake up time $t_{w}$ is trivially computed as

$$
\mathrm{t}_{\mathrm{w}}=\mathrm{t}_{\mathrm{A}}-\left(\mathrm{d}_{1}+\mathrm{d}_{2}+\ldots+\mathrm{d}_{\mathrm{n}}\right)
$$

The exact same formula can compute the wake-up time when we know when the person $\mathrm{P}$ arrived at the airport, and we know the durations of all the activities. For example, if we need to be at the airport at $2 \mathrm{pm}$, and the travel time from home is 20 minutes, then we need to leave home at 1:40 pm. Similarly, if we know that the person P arrived at the airport at $3 \mathrm{pm}$, and we know that it took him/her exactly 20 minutes to get there, this means that the person $\mathrm{P}$ left home at 2:40 pm - the same answer as for the previous problem.

Now consider the interval setting where $t_{A}$ and all $d_{i}$ are substituted by intervals, $T_{A}$ and $D_{i}$. Then the required wake up time $t_{w}$ is also trivially computed as $t_{w}=t_{A s}-\left(d_{1 e}+d_{2 e}+\ldots+d_{n e}\right)$, where $t_{A s}$ is the start point of the interval $T_{A}$, i.e., earliest desired arrival time, and each $d_{i e}$ is the endpoint of the respective interval $D_{i}$, i.e., longest time of each activity. This solution (wake up time $t_{w}$ ) can be called as the earliest pessimistic solution. It ensures that he will capture the plane with most pessimistic case when longest durations of all activities will happen. It is also the earliest among all pessimistic cases because it ensures the earliest arrival to the airport within the desired arrival interval $T_{A}$. Respectively the latest pessimistic solution is $t_{w}=t_{A e}-\left(d_{1 e}+d_{2 e}+\ldots+d_{n e}\right)$, where $t_{A e}$ is the end point of the interval $\mathrm{T}_{\mathrm{A}}$, i.e., latest desired arrival time. All other pessimistic solutions are between these two earliest and latest solutions.

Please note that for the modified airport task, the solution corresponding to interval uncertainty is different. For example, if we want to arrive not earlier than $2 \mathrm{pm}$ but not later than $3 \mathrm{pm}$, and the travel time takes between 20 and 40 minutes, then, to guarantee that we arrive exactly between 2 and $3 \mathrm{pm}$, we need to leave home between 1:40 pm and 2:20 pm. If we leave home before 1:40 pm, we run a risk of arriving too early (before $2 \mathrm{pm}$ ), and if we leave after 2:20 pm, we run a risk of arriving too late (after $3 \mathrm{pm}$ ). So, in this case, the solution to the original Airport task is the time interval $[1.40 \mathrm{pm}, 2.20 \mathrm{pm}]$. 
Let us now consider the modified task under the same interval uncertainty. Suppose that the person $\mathrm{P}$ arrives at the airport between $2 \mathrm{pm}$ and 3 pm, and we know that the travel time from home is between 20 and 40 minutes. We want to find out when the person $\mathrm{P}$ left home. Based on this information, the only thing we can conclude is that $\mathrm{P}$ left home between 1:20 pm (= $2 \mathrm{pm}-40$ minutes) and 2:40 pm (=3 pm -20 minutes). The resulting interval [1:20 pm, 2:40 pm] is exactly what interval computations predict - and it is different from the previous interval (that can be obtained, by the way, by using modal interval arithmetic; see, e.g., [Shary 1996]).

The bounds corresponding to the modified task are particular examples of what we call optimistic solutions. All optimistic solutions are between two earliest and latest optimistic solutions,

$$
\mathrm{t}_{\mathrm{w} 1}=\mathrm{t}_{\mathrm{As}}-\left(\mathrm{d}_{1 \mathrm{~s}}+\mathrm{d}_{2 \mathrm{~s}}+\ldots+\mathrm{d}_{\mathrm{ns}}\right), \mathrm{t}_{\mathrm{w} 2}=\mathrm{t}_{\mathrm{Ae}}-\left(\mathrm{d}_{1 \mathrm{~s}}+\mathrm{d}_{2 \mathrm{~s}}+\ldots+\mathrm{d}_{\mathrm{ns}}\right)
$$

We call these solutions optimistic because they assume shortest durations of all activities. The first one, $t_{\mathrm{w} 1}$, ensures the earliest optimistic wake up time $t_{w}$ because it uses the start point of the interval $\mathrm{T}_{\mathrm{A}}$. Similarly the second one, $\mathrm{t}_{\mathrm{w} 2}$, ensures the latest optimistic wake up time tw because it uses the end point of the interval TA.

All other solutions are between latest optimistic wake up time and earliest pessimistic wake up time. Note that optimistic solutions may not be appropriate solutions for the original task, because the person P may not be able to conduct activities with the shortest duration. For instance, traffic jam or an accident can prevent the person $\mathrm{P}$ from enjoying the shortest driving time.

Now we will represent concepts of interval equations and solutions in the formal terms including pessimistic and optimistic solutions using universal and existential quantifiers. Consider equation (2) where $\mathrm{A}, \mathrm{X}$ and $\mathrm{B}$ are intervals. For the airport task $\mathrm{X}$ is $\mathrm{T}_{\mathrm{w}}, \mathrm{B}$ is $\mathrm{T}_{\mathrm{A}}$, and all $\mathrm{D}_{\mathrm{i}}$ are the same as above.

$$
\mathrm{X}+\mathrm{D}_{1}+\mathrm{D}_{2}+\ldots \mathrm{D}_{\mathrm{n}}=\mathrm{B}
$$

There are four specifications of equation (2) [Sharyi, 1996, 2002; Horc1k, 2008] that are different tasks:

(S1) $\forall \mathrm{i} \forall \mathrm{d}_{\mathrm{i}} \in \mathrm{D}_{\mathrm{i}} \forall \mathrm{b} \in \mathrm{B} \exists \mathrm{x} \in \mathrm{X}: \mathrm{x}+\mathrm{d}_{1}+\mathrm{d}_{2}+\ldots+\mathrm{d}_{\mathrm{n}}=\mathrm{b}$

(S2) $\forall \mathrm{i} \forall \mathrm{d}_{\mathrm{i}} \in \mathrm{D}_{\mathrm{i}} \exists \mathrm{b} \in \mathrm{B} \quad \exists \mathrm{x} \in \mathrm{X}: \mathrm{x}+\mathrm{d}_{1}+\mathrm{d}_{2}+\ldots+\mathrm{d}_{\mathrm{n}}=\mathrm{b}$

(S3) $\forall \mathrm{i} \quad \exists \mathrm{d}_{\mathrm{i}} \in \mathrm{D}_{\mathrm{i}} \forall \mathrm{b} \in \mathrm{B} \exists \mathrm{x} \in \mathrm{X}: \mathrm{x}+\mathrm{d}_{1}+\mathrm{d}_{2}+\ldots+\mathrm{d}_{\mathrm{n}}=\mathrm{b}$

(S4) $\forall \mathrm{i} \quad \exists \mathrm{d}_{\mathrm{i}} \in \mathrm{D}_{\mathrm{i}} \exists \mathrm{b} \in \mathrm{B} \exists \mathrm{x} \in \mathrm{X}: \mathrm{x}+\mathrm{d}_{1}+\mathrm{d}_{2}+\ldots+\mathrm{d}_{\mathrm{n}}=\mathrm{b}$

\section{Pessimistic formulations}

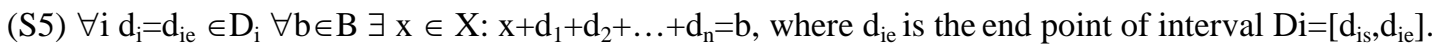
These are all pessimistic formulations.

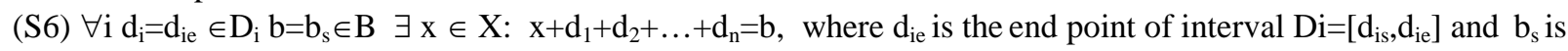
the start point of interval $b_{s} \in B=\left[b_{s}, b_{e}\right]$. This is the earliest pessimistic formulation.

(S7) $\forall \mathrm{i} \mathrm{d} \mathrm{d}_{\mathrm{i}}=\mathrm{d}_{\mathrm{ie}} \in \mathrm{D}_{\mathrm{i}} \mathrm{b}=\mathrm{b}_{\mathrm{e}} \in \mathrm{B} \quad \exists \mathrm{x} \in \mathrm{X}: \mathrm{x}+\mathrm{d}_{1}+\mathrm{d}_{2}+\ldots+\mathrm{d}_{\mathrm{n}}=\mathrm{b}$, where $\mathrm{d}_{\mathrm{ie}}$ is the end point of interval $\mathrm{Di}=\left[\mathrm{d}_{\mathrm{is}}, \mathrm{d}_{\mathrm{ie}}\right]$ and $\mathrm{b}_{\mathrm{e}}$ is the start point of interval $b_{s} \in B=\left[b_{s}, b_{e}\right]$. This is the latest pessimistic formulation.

\section{Optimistic formulations}

(S8) $\forall \mathrm{i} \mathrm{d}_{\mathrm{i}}=\mathrm{d}_{\mathrm{is}} \in \mathrm{D}_{\mathrm{i}} \forall \mathrm{b} \in \mathrm{B} \exists \mathrm{x} \in \mathrm{X}: \mathrm{x}+\mathrm{d}_{1}+\mathrm{d}_{2}+\ldots+\mathrm{d}_{\mathrm{n}}=\mathrm{b}$, where $\mathrm{d}_{\mathrm{ie}}$ is the end point of interval $\mathrm{Di}=\left[\mathrm{d}_{\mathrm{is}}, \mathrm{d}_{\mathrm{ie}}\right]$.

These are all optimistic formulations.

(S9) $\forall \mathrm{i} \mathrm{d}_{\mathrm{i}}=\mathrm{d}_{\mathrm{is}} \in \mathrm{D}_{\mathrm{i}} \mathrm{b}=\mathrm{b}_{\mathrm{s}} \in \mathrm{B} \quad \exists \mathrm{x} \in \mathrm{X}: \mathrm{x}+\mathrm{d}_{1}+\mathrm{d}_{2}+\ldots+\mathrm{d}_{\mathrm{n}}=\mathrm{b}$, where $\mathrm{d}_{\mathrm{ie}}$ is the end point of interval Di=[ $\left.\mathrm{d}_{\mathrm{is}}, \mathrm{d}_{\mathrm{ie}}\right]$ and $\mathrm{b}_{\mathrm{s}}$ is the start point of interval $b_{s} \in B=\left[b_{s}, b_{e}\right]$. This is the earliest optimistic formulation.

(S10) $\forall \mathrm{i} \mathrm{d} \mathrm{d}_{\mathrm{i}}=\mathrm{d}_{\text {is }} \in \mathrm{D}_{\mathrm{i}} \mathrm{b}=\mathrm{b}_{\mathrm{e}} \in \mathrm{B} \exists \mathrm{x} \in \mathrm{X}: \mathrm{x}+\mathrm{d}_{1}+\mathrm{d}_{2}+\ldots+\mathrm{d}_{\mathrm{n}}=\mathrm{b}$, where $\mathrm{d}_{\mathrm{ie}}$ is the end point of interval Di=[ $\left.\mathrm{d}_{\mathrm{is}}, \mathrm{d}_{\mathrm{ie}}\right]$ and $\mathrm{b}_{\mathrm{e}}$ is the start point of interval $b_{s} \in B=\left[b_{s}, b_{e}\right]$. This is the latest optimistic formulation.

As we can see the sum of intervals for X depends not only X,.but on B and all Di. It can be viewed as a form of a parametric sum. For comparison see [Popova, 2013] where parametric formulation and solution for the interval linear equations is proposed.

Conclusion from these ten formulations for the interval equation (2): 
1) In case of uncertainty, the equation (2) itself is incomplete, we need additional information to successfully solve the corresponding practical problem.

2) Each formulation S1-S10 added to Equation (2) produces a mathematically complete formulation that is sufficient for identifying if $\mathrm{X}$ is a solution or not and for designing an algorithm to find the solution $\mathrm{X}$.

3) Each formulation from S1-S10 of equation (2) produces its own set $X$ of solutions.

4) There is no room for the single interval arithmetic to solve equation (2). The single interval arithmetic would produce the same $\mathrm{X}$ for all formulations.

As it is easy to expect the same conclusions will be true if components of equation (2) are probabilities or fuzzy sets, because intervals are simplest forms of both of them. It was well recognized in the probability literature by noticing the need in additional information to make Eq. (2) mathematically complete. Examples of this information are knowledge from the specific task, different forms of regularization of the equation, and properties such as smoothness (see section 3). It was also recognized in the fuzzy systems literature long time ago [Dubois, Prade, 1984], but at the best of our knowledge was left mostly undeveloped. A single fuzzy arithmetic based in the Zadeh's extension principle continues to dominate in fuzzy systems literature while new developments beyond of it also started [Piegat, Pluciński, 2015]. The major message of this paper is that studies must expand beyond this narrow focus on a single type of fuzzy arithmetic, but to developing multiple fuzzy arithmetics derived from the real world tasks, not just postulated.

Now consider the interval setting with vector not scalar $\mathbf{X}$. Let, for instance each $\mathrm{x}$ consists of four variables that needs to be found, $\mathrm{x}=\left(\mathrm{x}_{1}, \mathrm{x}_{2}, \mathrm{x}_{3}, \mathrm{x}_{4}\right)$. In the original airport task formulation above we assumed that the person control only his wake up time, $\mathrm{x}$. In fact a traveler can control to some extent at least 4 variables: wake up time, $\mathrm{x}_{1}$, washing time, $\mathrm{x}_{2}$, eating breakfast time, $\mathrm{x}_{3}$, and departure time from home, $\mathrm{x}_{4}$.

Assume that he can control these uncertain times within respective intervals, $\mathrm{X}_{1}, \ldots, \mathrm{X}_{4}$. This means that in the equation $\mathrm{X}+\mathrm{D}_{1}+\mathrm{D}_{2}+\ldots+\mathrm{D}_{\mathrm{n}}=\mathrm{B}$ some $\mathrm{D}_{\mathrm{i}}$ are moving to $\mathrm{X}$. As before $\mathrm{B}$ is the desired arrival time at the airport time given as an interval time. This interval depends on the departure time and rather controlled by the airport and the airlines than by the passengers. Airlines commonly ask to be at the airport 2 hours before the departure time, $t_{d}$. Thus for this example a person can set up a desired $B$ as an hour length interval, $B=\left[t_{d}-2.5, t_{d}-1.5\right]$. In these terms in the interval formulation we need to solve the interval equation

$$
\mathrm{X}_{1}+\mathrm{X}_{2}+\mathrm{X}_{3}+\mathrm{X}_{4}+\mathrm{D}_{1}+\mathrm{D}_{2}+\ldots+\mathrm{D}_{\mathrm{k}}=\mathrm{B}
$$

Respectively task specifications S1-S10 for Eq (3) can be rewritten. Below we show them for S1-S4:

(S1) $\forall \mathrm{i} \forall \forall \mathrm{d}_{\mathrm{i}} \in \mathrm{D}_{\mathrm{i}} \forall \mathrm{b} \in \mathrm{B} \forall \mathrm{j} \exists \mathrm{x}_{\mathrm{j}} \in \mathrm{X}_{\mathrm{j}}: \mathrm{x}_{1}+\mathrm{x}_{2}+\mathrm{x}_{3}+\mathrm{x}_{4}+\mathrm{d}_{1}+\mathrm{d}_{2}+\ldots+\mathrm{d}_{\mathrm{k}}=\mathrm{b}$

(S2) $\forall \mathrm{i} \forall \mathrm{d}_{\mathrm{i}} \in \mathrm{D}_{\mathrm{i}} \exists \mathrm{b} \in \mathrm{B} \quad \forall \mathrm{j} \exists \mathrm{x}_{\mathrm{j}} \in \mathrm{X}_{\mathrm{j}}: \mathrm{x}_{1}+\mathrm{x}_{2}+\mathrm{x}_{3}+\mathrm{x}_{4}+\mathrm{d}_{1}+\mathrm{d}_{2}+\ldots+\mathrm{d}_{\mathrm{k}}=\mathrm{b}$

(S3) $\forall \mathrm{i} \exists \mathrm{d}_{\mathrm{i}} \in \mathrm{D}_{\mathrm{i}} \forall \mathrm{b} \in \mathrm{B} \forall \mathrm{j} \exists \mathrm{x}_{\mathrm{j}} \in \mathrm{X}_{\mathrm{j}}: \mathrm{x}_{1}+\mathrm{x}_{2}+\mathrm{x}_{3}+\mathrm{x}_{4}+\mathrm{d}_{1}+\mathrm{d}_{2}+\ldots+\mathrm{d}_{\mathrm{k}}=\mathrm{b}$

(S4) $\forall \mathrm{i} \exists \mathrm{d}_{\mathrm{i}} \in \mathrm{D}_{\mathrm{i}} \exists \mathrm{b} \in \mathrm{B} \quad \forall \mathrm{j} \exists \mathrm{x}_{\mathrm{j}} \in \mathrm{X}_{\mathrm{j}}: \mathrm{x}_{1}+\mathrm{x}_{2}+\mathrm{x}_{3}+\mathrm{x}_{4}+\mathrm{d}_{1}+\mathrm{d}_{2}+\ldots+\mathrm{d}_{\mathrm{k}}=\mathrm{b}$

The Eq (3) presents an interesting case that links interval and probabilistic formulations. While in the interval formulation, all points in all $X_{i}$ and $D_{j}$ are considered as equally possible/probable their sums have different frequencies. In probabilistic formulation if all $X_{i}$ and $D_{j}$ represent independent uniform pdfs then their sum will be a unimodal pdf. This means that different sums $\{b\}$ have different probabilities. Respectively some sum $b_{1}$ can have much larger set of solution vectors $\left\{\left(\mathrm{x}_{1}, \mathrm{x}_{2}, \mathrm{x}_{3}, \mathrm{x}_{4}\right)\right\}$ than another sum $\mathrm{b}_{2}$. Selecting $\mathrm{b}_{1}$ with greater probability value gives him potentially more options for wake up time, duration of breakfast and other activities that he can control. This leads to another task specification S11:

(S11) $\forall \mathrm{i} \forall \mathrm{d}_{\mathrm{i}} \in \mathrm{D}_{\mathrm{i}} \forall \mathrm{b} \in \mathrm{B} \forall \mathrm{j} \exists \mathrm{x}_{\mathrm{j}} \in \mathrm{X}_{\mathrm{j}}: \mathrm{x}_{1}+\mathrm{x}_{2}+\mathrm{x}_{3}+\mathrm{x}_{4}+\mathrm{d}_{1}+\mathrm{d}_{2}+\ldots+\mathrm{d}_{\mathrm{k}}=\mathrm{b} \& \mathrm{~b}=\arg \left(\max f\left(\mathrm{~b}_{\mathrm{r}}\right)\right)$,

where $f\left(b_{k}\right)$ is a pdf for all possible sums $b_{r}$ in B.

S11 also can be modified to produce pessimistic solutions similar to S5-S7 above. In the case of S5 we will get a widest set of options to select values of variables that he can control from a variety of wake up times to different duration of breakfast and times to leave home under the assumption that uncontrolled variables will have their longest durations (pessimistic assumption). 


\subsection{Probabilistic Settings}

Now consider the probabilistic settings where in Eq (3) all $X_{i}, D_{k}$, and $B$ are given with pdfs. As we already stated Eq (3) provides an incomplete mathematical formulation and needs to be augmented with specifications. We will continue to analyze the airport task for this case.

The probabilistic settings with different specifications produce different pdfs for B. At this moment we will concentrate not on these differences, but on types of tasks that can be formulated having a pdf. The most obvious one is a search for the solution that maximizes this pdf as was the case in S11 above.

What is the meaning of this solution in general - and, in particular, for the original airport task? To clarify this we need first to analyze the meaning of the pdfs for $X_{i}$ and $D_{j}$. The traveler cannot influence and ignore pdfs for all $D_{j}$, They must be taken into account. The situation with $\mathrm{X}$ is different. Consider the breakfast duration which is from $\mathrm{X}$. For instance, it can have a unimodal pdf on interval from 10 to 20 minutes with mean 15 minutes. This pdf can be ignored and the traveler can use any time from this interval if needed. Respectively maximization of probabilities can be done only using probabilities coming from all $\mathrm{D}_{\mathrm{j}}$. In this case the solution with max of pdf, max $f\left(\mathrm{~b}_{\mathrm{r}}\right)$, will be the solution that uses most probable values of variables that are not controlled by the travelers such as duration of driving to the airport (due to traffic pattern). This solution has an obvious drawback-it does not guarantee him catching the plane. This strategy will work only statistically when a traveler is interested to catch multiple planes say over the year with highest probability. This is not the case in the task when he needs to catch for sure the specific plane at the given date. In this case he would need to use some solutions of the pessimistic task specification and max of pdf can be an additional requirement to the pessimistic task specification. As a "pessimistic" solution this solution does not use pdfs for $\mathrm{D}_{\mathrm{i}}$, but worst cases of all $\mathrm{D}_{\mathrm{i}}$.(longest durations). If also X pdfs are ignored then it becomes the interval based formulation that we already discussed above. In both cased if X pdfs are not ignored or ignored the max of pdf on B will gives a solution with the largest number of alternative solutions that ensure that the traveler will not miss his plane.

\subsection{Fuzzy sets settings}

Now consider the fuzzy sets settings where in Eq (3) all $X_{\mathrm{i}}, \mathrm{D}_{\mathrm{k}}$, and $\mathrm{B}$ are given as fuzzy sets /fuzzy numbers. The Zadeh's extension principle is doing exactly the same as max of pdf in the probabilistic setting, but for membership functions of fuzzy sets. It does not guaranty that the traveler will catch his plane without requiring a pessimistic specification of the solution. Under this requirement the extension principle will provide solutions that maximize the membership function, $m\left(b_{r}\right)$ that represents sums $x_{1}+x_{2}+x_{3}+x_{4}+d_{1}+d_{2}+\ldots+d_{k}=b$. The meaning of this maximization will depend on definition of $m\left(\mathrm{~b}_{\mathrm{r}}\right)$.

If $m\left(\mathrm{~b}_{\mathrm{k}}\right)$ is a probability-based function [Kovalerchuk, 2014, 2015] then it is in essence the same as it is in the probabilistic setting discussed above. If it is a possibilistic setting then it is less clear because the possibilistic interpretation has no operational definition as this concept is defined by Bridgeman [1927] in his operationalism theory.

The optimistic solution in probabilistic and fuzzy sets settings means respectively that we will get a solution with the highest probability or membership value. Similarly pessimistic solutions mean getting the lowest probability and membership values. In these terms the extension principle is optimistic. However in the probabilistic interpretation of the membership function it may or may not be optimistic in probabilistic sense. The reason in that it is an upper estimate of the probability and it is not necessary that upper estimate for the max of the probability will get the upper estimate higher than for other alternatives. Fuzzy extension principle is not an optimistic estimate for the probability, but rather its upper estimate that may never be reached.

The important point in this analysis is that abstract sum of fuzzy sets $X_{1}+X_{2}+X_{3}+X_{4}$-- defined without context of this task -- will be useless for solving this task. Unfortunately such practice still continues in some works. Next solution of (1) is not a solution of the catching the plane task. It only gives the constraint. We need an optimization objective function, or a set of objective functions in a multi-objective optimization setting. 


\section{Lessons from prior studies}

\subsection{Defining solution for fuzzy differential equations}

As we already stated in the introduction most of the activities in the fuzzy systems for fuzzy equations were concentrated on finding solutions and conditions when solutions exist under the definition of solution based on Zadeh's extension principle. The paper by Buckley, Feuring [2000] is representative sample of this type of studies for both algebraic and differential equations. They start from the first-order ordinary differential equation

$$
\mathrm{d} y / \mathrm{d} t=f(t, y, k), y(0)=\mathrm{c}
$$

where $\mathrm{k}=\left(\mathrm{k}_{1}, \ldots, \mathrm{k}_{\mathrm{n}}\right)$ is a vector of constants, and $\mathrm{t}$ is in some closed and bounded interval, which contains zero. It is assumed that $f$ satisfies some conditions so that (I) has an unique crisp solution $y=g(t, k, c)$, for $t \in l, k \in K \subset R^{\mathrm{n}}$, $c \in C \subset R$.

The fuzzification is defined by introduction of a vector of triangular fuzzy numbers $K^{\wedge}=\left(K^{\wedge} \wedge_{\mathrm{i}}, \ldots, K^{\wedge}{ }_{\mathrm{n}}\right)$ and another triangular fuzzy number $C^{\wedge}$. Then $\mathrm{k}$ is substituted by $K^{\wedge}$ and $\mathrm{c}$ is substituted by $C^{\wedge}$ in (I) to get

$$
\mathrm{d} Y \wedge / \mathrm{d} t=f\left(t, Y^{\wedge}, K^{\wedge}\right), \quad f(0)=C^{\wedge}
$$

under the assumption of some definition for the derivative of fuzzy function $f\{t$ ) from the literature and that $f$ is a fuzzy number for each $t$ in $I$.

Solving (5) is defined as finding $Y^{\wedge}(t)$ that itself is defined in three equivalent ways:

(W1) by fuzzification of the crisp solution $y=g(t, k, c)$ using the extension principle to get $Y^{\wedge}(\mathrm{t})=g\left(t, K^{\wedge}, C^{\wedge}\right)$

(W2) by using $\alpha$-cuts $\mathrm{K}(\alpha)=\mathrm{K}^{\wedge}{ }_{1}[\alpha] \times \cdots \times \mathrm{K}^{\wedge}{ }_{\mathrm{n}}[\alpha]$ and $\Phi(\alpha)=\mathrm{K}(\alpha)$ x $\mathrm{C}[\alpha]$, for $0 \leq \alpha \leq 1$ to get $\alpha$-cuts

where

$$
Y(t)[\alpha]=\left[Y_{1}(t, \alpha), Y_{2}(t, \alpha)\right]
$$

$$
\begin{aligned}
& Y_{1}(t, a)=\min \{g(t, k, c) \mid \mathrm{k} \in \mathrm{K} \wedge[\mathrm{a}], \mathrm{c} \in \mathrm{C}[\mathrm{a}]\} \text { and } \\
& y_{2}(t, a)=\max \{g(t, k, c) \mid \mathrm{k} \in \mathrm{K} \wedge[\mathrm{a}], \mathrm{c} \in \mathrm{C}[\mathrm{a}]\},
\end{aligned}
$$

(W3) by using $\boldsymbol{\alpha}$-cuts $\Omega(\alpha)=\{g(t, k, c) \mid(\mathrm{k}, \mathrm{c}) \in \Phi(\alpha)\}$ for $0 \leq \alpha \leq 1$, and $\boldsymbol{t} \in \boldsymbol{I}$ as the membership function

$$
\mathrm{Y}^{\wedge}(\mathrm{t})(\mathrm{x})=\sup \{\alpha \mid \mathrm{x} \in \Omega(\alpha)\}
$$

These authors gave necessary and sufficient conditions for $\mathrm{Y}(\mathrm{t})$ to solve this fuzzy initial value problem. The extension to fuzzy partial differential equations is proposed in the same way as finding the crisp solution, fuzzifying it and then checking to see if it satisfies the fuzzy partial differential equation. As we see the extension principle is accepted without discussing the justification of definition of the solution based on the extension principle.

\subsection{Systems of stochastic linear algebraic equations}

Girko in a series of publications [1992, 1996, 1998] discussed solutions for a system of linear algebraic equations (SLAE) Ay=b, when their coefficients A and b are given with some random errors. He pointed out [Girko, 1992] that "it is not yet clear how to find the best consistent, in some sense, estimates of the solutions of SLAE, if their coefficients are given with some random errors, and conditions of existence of moments of the components $\mathrm{y}_{\mathrm{k}}$ of the vector y have not been found."

He provides an equation (6) presented below, where $\mathrm{A}$ is a matrix of order $\mathrm{n} \times \mathrm{m}$ and a transposed matrix is denoted by a "prime". The expression (6) is called a regularized pseudo-solution of SLAE Ay=b with random coefficients and nonsingular matrices $C_{1}, C_{2}$ are that provide regularization, where $\alpha \geq 0, \beta>0$ vare some constants,

$$
y_{\alpha}=\left(C_{1}^{\prime} C_{1} \alpha+A^{\prime}\left(C_{2}^{\prime}\right)^{-1} C_{2}^{-1} A \beta^{-1}\right)^{-1} A^{\prime}\left(C_{2}^{\prime}\right)^{-1} C_{2}^{-1} \beta^{-1} b
$$


His main focus was on the mathematical issues of funding approximation of solution (6) for A with a large n. Our focus is on the question of the justification of a definition of the solution. This regularized solution needs to be justified for each applied task that includes justification of $\alpha, \beta, C_{1}$ and $C_{2}$. This question was left unanswered in that work.

Provencher [1982] pointed out that the problems of stochastic linear equations generally have a large number of possible solutions (the ill-posed inversion problem) with arbitrary large deviations from each other all of which fit to the error distribution functions obtained experimentally. Kac [1943] studied the average number of real roots of a specific random algebraic equation $X_{0}+X_{1} X+X_{2} X^{2}+\ldots+X_{n-1} x^{n-1}=0$, where the $X$ 's are independent random variables with the same and particular normal distribution. This was an extension of the previous studies conducted by Littlewood and Offord. As we see it is not a general linear stochastic equation, but a quite specific one that allowed estimating the average number of solutions.

Respectively Provencher [1982] stated "straightforward inversion procedures cannot be used and statistical regularization techniques are necessary". Then he discusses most relevant for us the issue of selecting and justifying regularization method. The idea is to find a simplest solution that is consistent with prior knowledge and experimental data that can be available in addition to the stochastic equation. While simplicity can be achieved and was achieved by multiple methods including listed in [Provencher, 1982], its relevance to the task at hand is not obvious. In the common approach the regularizer will impose simplicity (typically smoothness) or statistical prior knowledge. The search for such simplest regularizers can be conducted by solving a quadratic optimization problem and by using F-text and confidence regions. Methods of explicit solving stochastic systems of linear algebraic equations that include the Monte Carlo method, the perturbation method, the Neumann expansion method and the polynomial chaos have been reviewed in [Li at al, 2006].

In essence simplicity is an external criterion to the task at hand. For our airline task experimental data on the duration of breakfast and driving to the airport will likely shift the solution to the averages of these values not to the pessimistic durations that will guaranty that the plane will not be missed.

\subsection{Stochastic programming}

As we see the regularization actually converts solving the stochastic equation/equations to solving an optimization task where the original equation/equations can be modified or used as constraints. The advantage of explicitly stated optimization task is that it allows clearly separate technical external to the task assumptions from ones derived from the task and relevant to the domain knowledge. The same is applicable for solving fuzzy equations by solving fuzzy optimization tasks. The assumption of fuzzy optimization tasks have been reviewed in [Kovalerchuk, 1994] and seem still valid.

For instance in the classical two-stage linear stochastic programming problems [Shapiro et al, 2009, King,Wallace, 2012] it is clearly stated that at the first stage we minimize the cost of the first-stage decision plus the expected cost of the second-stage decision. It is assumed that the second-stage cost is a random vector with a known probability distribution. It means that we deal with randomness of the second-stage cost by minimizing its average (expected cost). If for a particular task this is not appropriate like in our airline task the objective function must be rewritten appropriately.

\section{Conclusion}

In many practical situations, we need to solve equations and systems of equations under uncertainty. This uncertainty can be interval, probabilistic, fuzzy, etc. To solve such problems, at first glance, it seems reasonable to take the solution to the corresponding exact systems and apply the general translation to the corresponding type of uncertainty - interval computations for interval uncertainty, extension principle for fuzzy uncertainty, etc. And this is exactly how many researchers and practitioners often solve the corresponding uncertain problems. In this paper, we emphasize that the resulting solutions are sometimes inadequate.

The reason for this inadequacy is that to come up with a correct solution, we need to analyze the original problem: because several different practical problems -- which lead to the same solution for exact data - can lead to completely different solutions in the presence of uncertainty. This ambiguity cannot be resolved by simply 
modifying the usual formal approach: e.g., even for the simple case when the exact-case solution is just a subtraction, in the uncertainty case, there can be at least ten different definitions of a solution.

Our recommendation to always take into account the meaning of the corresponding problem - as opposed to just the equations - is in perfect accordance with the original spirit of fuzzy systems approach: that, to properly analyze reallife systems, we need to take into account not only the corresponding equations, but also the experts' knowledge that goes beyond these equations.

\section{References}

1. Bridgman, P.W., The Logic of Modern Physics, Macmillan, New York, 1927.

2. $\quad$ Buckley, J., Feuring. T., Fuzzy differential equations, Fuzzy Sets and Systems 110 (2000) 43-54

3. Girko V.L., Systems of Linear Algebraic Equations with Random Coefficients, Theory of Probability \& Its Applications, 36(2), $261-271$. SIAM, 1992, DOI:10.1137/1136030

4. Girko V.I. Theory of Linear Algebraic Equations with Random Coefficents, N.Y., 1996

5. Girko V.I., An Introduction to Statistical Analysis of Random Arrays, VSP, 1998

6. Di Nola, A., Pedrycz, W., Sessa, S. On Measures of Fuzziness of Solutions of Fuzzy Relation Equations with Generalized Connectives, Journal of Mathematical Analysis and Applications 106, 443-453, 1985

7. $\quad$ Dubois, D., Prade, H., Fuzzy-set-theoretic differences and inclusions and their use in the analysis of fuzzy equations, Control and Cybernetics 13(3) (1984) 129--145.

8. Gottwald S. Generalized solvability behaviour for systems of fuzzy equations, in: Discovering the world with fuzzy logic, Physica Verlag, Heidelberg, 2000, 401-430.

9. Hisdal. E., Are grades of membership probabilities' Fuzzy Sets and Systems 25 (1988) 325-348.

10. Horcik, R., Solution of a system of linear equations with fuzzy numbers, Fuzzy Sets and Systems, Vol. 159, pp. 1788-1810, 2008

11. Kac M., On the average number of real roots of a random algebraic equation, Bull. Amer. Math. Soc. 49(4) (1943) 314-320.

12. King, A.; Wallace, S., Modeling with Stochastic Programming. Springer Series in Operations Research and Financial Engineering, Springer, New York, 2012

13. Kovalerchuk, B. Summation of Linguistic Numbers, Proc. of North American Fuzzy Information Processing Society (NAFIPS) and World Congress on Soft Computing, 08-17-19, 2015, Redmond, WA pp.1-6. DOI: 10.1109/NAFIPS-WConSC.2015.7284161

14. Kovalerchuk, B., Probabilistic Solution of Zadeh’s test problems, In: A. Laurent et al. (Eds.): IPMU 2014, Part II, CCIS 443, pp. 536-545, 2014, Springer.

15. Kovalerchuk, B. Current situation in foundations of fuzzy optimization. In: M. Delgado, J. Kacprzyk, J. L. Verdegay and M.A. Vila (Eds.): Fuzzy Optimization: Recent Advances, Studies in Fuzziness, Springer, Heidelberg, NY, 1994, pp.45 60.

16. Kreinovich, V. Solving equations (and systems of equations) under uncertainty: how different practical problems lead to different mathematical and computational formulations, Granular Computing, to appear.

17. Li, C.F., Feng, Y.T., Owen, D.R.J., Explicit solution to the stochastic system of linear algebraic equations (a1A1 + a2A2 +... amAm)x = b, Comput. Methods Appl. Mech. Engrg. 195 (2006) 6560-6576.

18. Peeva K., Fuzzy Linear Systems, Annales Univ. Sci. Budapest., Sect. Comp. 12 (1991) 201-207.

19. Pellissetti, M.F. , Ghanem R.G. , Iterative solution of systems of linear equations arising in the context of stochastic finite elements, Advances in Engineering Software 31 (2000) 607-616.

20. Piegat A., Pluciński, M., Fuzzy Number Addition with the Application of Horizontal Membership Functions, The Scientific World Journal Volume 2015 (2015), Article ID 367214, 16 pages http://dx.doi.org/10.1155/2015/367214

21. Popova, E.D., Improved enclosure for some parametric solution sets with linear shape, Computers and Mathematics with Applications 66 (2013) 1655-1665. http://dx.doi.org/10.1016/j.camwa.2013.04.007

22. Provencher S., CONTIN: a general purpose constrained regularization program for inverting noisy linear algebraic and integral equations, Computer Physics Communications, 27 (1982) 229-242.

23. Sanchez. E., Solution of fuzzy equations with extended operations. Fuzzy Sets and Systems, 12:237-248, 1984.

24. Shary, S. P., A new technique in systems analysis under interval uncertainty and ambiguity. Reliable Computing, 8:321-418, 2002.

25. Shary, S.P. Algebraic approach to the interval linear system identification, tolerance, and control problems, or one more application of Kaucher arithmetic, Reliable Computing, 2(1) (1996) 3-33.

26. Shapiro, A; Dentcheva, D., Ruszczyński, A (2009). Lectures on stochastic programming: Modeling and theory. MPS/SIAM Series on Optimization 9. Philadelphia, PA: pp. xvi+436.

27. Skalna, M.V. Rama Rao, Andrzej Pownuk, Systems of fuzzy equations in structural mechanics, Journal of Computational and Applied Mathematics 218 (2008) 149 - 156

28. Yager R., On Solving Fuzzy Mathematical Relationships, Information and Control, 41 (1979) 29-55. 